\title{
«Solo un'immensa fonte di dolore». Appunti per una ricerca sulle donne in manicomio durante la Grande guerra
}

ISSN: 2282-4979

DOI: 10.12977/ereview55

Questo articolo è stato sottoposto a un processo di double blind peer review

Questo articolo si occupa di donne ricoverate negli ospedali psichiatrici dell'Emilia Romagna durante la Prima guerra mondiale, ma anche delle donne che vi lavorarono, come infermiere e come medici. Il punto di partenza sono stati i gravi cambiamenti che il conflitto portò anche nella vita delle donne (problemi economici, separazioni familiari, lutti). Da qui sono sorti alcuni quesiti: che conseguenze ebbero questi disagi cosi prolungati sui nervi e sulle menti di madri, mogli, figlie? Quante donne accusarono problemi tali da portarle al ricovero in manicomio?

This article deals with women admitted to psychiatric hospitals of the Emilia Romagna during the First World War, but also of the women who worked there, such as nurses and doctors. The starting point were the major changes that the conflict led in the lives of women (economic problems, family breakdown, mournings). Hence some questions arise: what consequences these discomforts had on the nerves and on the minds of mothers, wives, daughters? How many women accused problems that brought them to the asylum?

\section{Premessa}

La Prima guerra mondiale, guerra davvero «totale», rappresentò uno sconvolgimento tale da coinvolgere concretamente ogni aspetto della vita di di uomini e donne, bambini e adulti, militari e civili. Una guerra che costrinse non solo i combattenti, ma anche la popolazione civile a fare esperienze nuove e inattese, dolorose e traumatiche. Un trauma immane che non risparmiò, ma anzi infierì anche sui nervi e sull'equilibrio psichico dei militari, compromettendone in molti casi l'esistenza e 
lasciandoli invalidi o «mutilati dell'anima», per riprendere l'espressione di Gaetano Boschi'. Sulla cosiddetta "psichiatria di guerra", sulle menti traumatizzate dagli assalti e dai bombardamenti, è stato scritto moltissimo, anche di recente, e in particolare sul caso italiano ${ }^{2}$. È stato calcolato che solo fra i militari italiani furono almeno 40.000 gli uomini finiti in manicomi durante il conflitto; anche a causa dell'arrivo di questa massa di ricoverati, gli ospedali psichiatrici del nostro Paese furono letteralmente "travolti" dalle conseguenze della guerra. E così avvenne anche per i manicomi emiliano-romagnoli. D'altra parte, molti psichiatri e infermieri furono richiamati sotto le armi, in molti casi per essere impiegati nel Servizio neuro-psichiatrico allestito dell'esercito', che aveva il compito di gestire le prime fasi di assistenza per i militari che accusavano sintomi neuro-psichiatrici e inviarli, se ritenuto necessario, nei reparti psichiatrici militari nelle retrovie. Ciò comportò ovviamente gravi problemi di gestione nei manicomi, con carenze nel personale che fecero peggiorare le condizioni di vita già precarie degli internati.

Non va dimenticato in terzo luogo che per tanti psichiatri e psicologi italiani - da Giulio Cesare Ferrari a Placido Consiglio, da Ferdinando Cazzamalli a padre Agostino Gemelli - la Grande guerra fu anche l'occasione per mettere alla prova le proprie conoscenze e le proprie tecniche terapeutiche e di selezione (in primo luogo per scovare i simulatori). La guerra fu vista dalla classe alienistica italiana non soltanto come una sciagura, anche e soprattutto dal punto di vista eugenetico ${ }^{4}$, ma anche come un immenso laboratorio, da cui trarre importanti indicazioni per possibili strumenti di gestione della società anche in tempo di pace, per lottare più efficacemente contro tutta una serie di degenerazioni, anormalità, comportamenti devianti.

Qui noi ci occuperemo soltanto di una parte limitata, e non la più visibile senza dubbio, della vicenda complessiva dei manicomi italiani nella Prima guerra mondiale. $\mathrm{Ci}$ interessa in questa sede compiere soltanto una prima rassegna, di quanto già è stato scritto sulle presenze femminili nei manicomi negli anni della Grande guerra, riferendoci in particolare ai manicomi dell'Emilia-Romagna. Il passo successivo dovrà essere quello 
di dedicarsi alle cartelle cliniche e agli altri materiali conservati negli archivi degli ex-ospedali psichiatrici. In una formula, occorre mettersi a scavare nei registri di ammissione e fra le cartelle cliniche. E non soltanto a quelle delle donne ricoverate negli anni del conflitto o nell'immediato dopoguerra, ma anche a quelle dedicate proprio ai militari. Si potrebbero così far emergere anche un punto di vista femminile (delle donne in quanto madri, sorelle, figlie, mogli di militari ricoverati), così come esso appare, per esempio, nelle lettere che venivano inviate ai malati o agli psichiatri che li avevano in cura 5

\section{Donne mobilitate}

Fare la guerra è, da sempre, una cosa da uomini, un fenomeno essenzialmente maschile. L"“officina della guerra", il grande laboratorio della Grande guerra, rappresentò però uno sconvolgimento (temuto o ambito, a seconda dei punti di vista, piuttosto che concretamente realizzato), anche nei ruoli di genere, nella "gerarchia" fra uomo e donna ${ }^{6}$. Ad ogni modo, il dopoguerra avrebbe ben presto rimesso le cose a posto, riaffermando e rafforzando le differenze tradizionali: gli uomini al lavoro e impegnati semmai nella vita pubblica; le donne a casa.

Eppure un certo sconvolgimento, dentro il più grande sconvolgimento bellico, in effetti ci fu. E in particolare per un Paese per tanti versi arretrato come l'Italia. Le donne furono costrette talvolta a sostituire i lavoratori costretti al fronte, e furono soprattutto impegnate in ruoli di cura, di maternage (le "crocerossine"). La guerra rappresentò in questo senso il massimo livello di separazione: uomini e donne tenuti lontano coattivamente.

Divenne un luogo comune l'idea secondo la quale le donne, o almeno

Le cartelle cliniche rappresentano anche per questo tema una fonte di prima importanza, da mettere accanto ad altre forme di scrittura, privata e burocratica: «L'esplorazione del vissuto quotidiano, la valutazione "in soggettiva" dell'impatto dell'evento bellico sono state compiute anche grazie al mutamento delle fonti utilizzate dall'analisi storica; la guerra agì infatti come "moltiplicatore" delle forme della scrittura privata e burocratica; anche le donne, come i soldati, si avvicinarono alla scrittura per avere notizie dei propri cari, per compilare moduli di sussidio e richieste di licenze, per fermare sulla carta momenti drammatici: istanze, lettere, diari e memorie scritte, unite alle ultime testimonianze orali, sono quindi diventate fonti preziose e indispensabili» [Ermacora 2006, 14-5].

«L'idea che la Grande Guerra abbia profondamente trasformato il rapporto tra i sessi, ed emancipato le donne in misura molto maggiore dei precedenti anni, o persino secoli, di lotte, è assai diffusa durante e dopo il conflitto. È questo un luogo comune della letteratura e della politica, sia che questa frattura venga considerata positivamente o stigmatizzata, sia nel caso in cui è analizzata nella sua portata reale o esageratamente amplificata» [Thébaud 1992, 25]. 
alcune categorie di donne, avessero finito per assumere ruoli e comportamenti apertamente maschili. In questo senso, si impose l'immagine della "maschietta". Uomini e donne furono costretti, come vedremo, ad assumere caratteri propri dell'altro sesso. Le donne rimanevano sì nell'immaginario collettivo anzitutto come madri, mogli o fidanzate di militari. Ma si verificò la necessità di una momentanea emancipazione di tante donne, soprattutto di quelle appartenenti ai ceti popolari. La mobilitazione fu anche una "questione femminile", anche se non riuscì a mettere radicalmente in crisi la subalternità delle donne'. Donne in fabbrica, donne alla guida di tram, donne negli uffici: tutte immagini che rompevano con l'idea di un "mestiere femminile", e tutte immagini che avrebbero però finito per nuocere a una reale, duratura emancipazione, riconducendo questi progressi a una ruolo ausiliario e supplente. Questi cambiamenti riguardarono principalmente il campo lavorativo, ma non soltanto. Fu con la guerra che si ruppe l'incompatibilità fra donne e sfera pubblica [Di Cori 1986]. Fin dall'entrata in guerra, anche in Italia ci fu un ampio ricorso alla mobilitazione femminile. La storiografia si è concentrata soprattutto sul volontariato femminile nel fronte interno: un po' ovunque nel Paese crebbero e si svilupparono comitati locali, enti semi-pubblici a cui furono demandate funzioni di assistenza e di controllo sociale, e in cui donne appartenenti ai ceti medio-alti, alle élites intellettuali, poterono concretizzare il loro patriottismo. Pur evitando ogni generalizzazione, rimane fuori di dubbio che le "crocerossine" e le "madrine di guerra" divennero protagoniste nella vita quotidiana del fronte interno. Lavoravano per preparare bende, raccogliere lana, predisponevano raccolte di fondi, davano assistenza alle famiglie dei militari, organizzavano asili nido per le madri costrette a lavorare fuori casa, si prendevano cura di orfani di guerra.

Davanti alla necessità di curare masse di feriti, di mutilati, di moribondi, la mobilitazione delle donne fu vista come un allargamento del loro ruolo di cura all'intero corpo della nazione. Per questo le "crocerossine" furono essenziali per riconfermare la stabilità dei ruoli di genere, per rassicurare i soldati.

Questo tipo di impegno, però, rappresenta soltanto una parte della que-

«Molti storici e storiche hanno concluso che le guerre hanno una capacità limitata di emancipare le donne. Se aprono nuove possibilità, in genere si tratta di ruoli temporanei. Le guerre possono dare l'impressione di sowvertire l'ordine di genere, ma con la pace esso viene restaurato, sovente con veemenza. II tempo di guerra spesso porta con sé un'esaltazione dei tradizionali ruoli di genere, gli uomini sono ritratti come guerrieri e le donne come madri, custodi simboliche della pace, della normalità e del focolare domestico, che i soldati proteggono combattendo e al quale possono tornare al termine del conflitto» [Willson 2010, 73]. 
stione. Accanto alle infermiere e alle altre volontarie, si impose, fino a divenire un luogo comune, l'immagine della donna operaia, delle lavoratrici che sostituivano gli uomini al fronte. Non si trattò di un ingresso in masse nelle officine, anzi. La manodopera femminile modificò soprattutto l'immaginario [Gibelli 2005, 68]. Secondo Barbara Curli, la sostituzione delle donne durante la guerra fu un «mito» [Curli 1998, 13]. Si trattò di una minoranza significativa, che contribuì a ridefinire una cittadinanza femminile. Raramente, e solo in poche mansioni, le donne sostituivano direttamente gli uomini. Ciò avvenne soprattutto in campagna.

Ben poche sono le informazioni di cui si dispone sull'esperienza di guerra delle donne dei ceti subalterni. In particolare delle donne contadine. Quelle che, più di altre, ebbero la vita "invasa" dalla guerra. Non solo perché la guerra allontanò gran parte degli uomini dalle campagne, ma perché "invase" mondi dove più rigide erano le gerarchie patriarcali e più stabili i ruoli dei sessi [Molinari 2008, 56].

Nei contesti urbani, ciò che si verificò fu sostanzialmente un aumento di donne nelle occupazioni impiegatizie [Curli 1998, 20] e in settori limitati come il trasporto pubblico e i locali pubblici. Mansioni comunque ben visibili. Più problematico l'ingresso delle donne in fabbrica. Spesso guardate con diffidenza dai colleghi maschi, che potevano temere la concorrenza femminile, ma che ancora di più scontavano l'atavico pregiudizio verso la naturale debolezza e verso l'inadeguatezza delle donne al lavoro. Rimanevano comunque "mezzi uomini”.

Molto interessante notare che la Grande guerra fu al contempo un momento epocale che mise in crisi proprio la virilità degli uomini, dei soldati. Di fronte alla mascolinizzazione di operaie e contadine, ecco la femminilizzazione degli uomini.

Indubbiamente la Grande guerra è per gli uomini un lungo trauma, massacro, di massa, caricatura mortificante dell'immagine della guerra virile e trionfale, negazione di tutti i valori della cultura occidentale. Immobili, sprofondati nel fango e nel sangue delle trincee, condannati ad aspettare il momento mortale dell'assalto o il tiro dei cannoni nemici, vittime talvolta di malattie femminili come l'isteria catalogata dai medici inglesi con il nome di shell shock - i combattenti avvertono il senso di una regressione allo stato selvaggio e vivono la guerra come un'impotenza pubblica e privata. Quando essi correvano all'assalto del nemico, le donne aspettavano, religiosamente. Ora che, in loro assenza, esse accedono allo spazio e alle responsabilità pubbliche per far girare la macchina della guerra, hanno paura di essere spossessati e traditi [Thébaud 1992, 43-4].

Soffermiamoci su quest'ultimo punto, sulla donna "angelo del focolare". La guerra fu un momento critico per milioni di famiglie, e per milioni di relazioni affettive. Per tante ragioni, l'esperienza delle donne, di coloro 
che restavano a casa, dovendosi anche assumere il carico di una famiglia, fu anzitutto l'esperienza di una perdita, di un abbandono. Si perdevano, temporaneamente o per sempre, i mariti, i fratelli, i figli. Si perdevano il reddito, la sicurezza. Si entrava in un lungo, indefinito periodo di solitudine, di attesa, di sospensione angosciosa. L'attesa di notizie dal fronte, come l'attesa di un ritorno a casa (magari solo momentaneo, per una licenza), era appunto una condizione estenuante, snervante, che poteva senza dubbio rappresentare l'origine di una profonda sofferenza. Basta pensare alla tragica scena raccontata da Emilio Lussu in Un anno sull'altopiano. Dopo anni di guerra, Lussu torna per una breve licenza a casa, in Sardegna. Al momento della partenza, la madre, che aveva ostentato tranquillità, cade in una condizione di disperazione assoluta:

Al centro della sala, accanto a una sedia rovesciata, la mamma era accasciata sul pavimento, in singhiozzi. Io la raccolsi, l'aiutai a sollevarsi. Ma non si reggeva più da sola, tanto, in pochi istanti, si era disfatta. Tentai di dirle parole di conforto, ma si struggeva in lacrime [Lussu 1960, 164].

Poteva giungere da un momento all'altro la notizia di un lutto, temuto per mesi o per anni; e ciò poteva essere l'occasione traumatica in cui tante ansie che si erano accumulate esplodevano in crisi nervose. D'altra parte, anche chi aveva la fortuna di poter riabbracciare i figli, il marito, il padre, doveva affrontare il trauma del loro ritorno; il trauma del ritorno di uomini profondamente cambiati, a loro volta traumatizzati, dalla vita di trincea, dall'esperienza di una morte di massa, oppure feriti, mutilati, ciechi. Senza dubbio, fu il lutto l'esperienza più forte che colpì le donne, tanto forte da essere difficilmente comunicabile [Audoin-Rouzeau e Becker 2002]. Ciò contribuì a riaffermare, a ribadire il ruolo tradizionale delle donne, prima di tutto ed essenzialmente come madri. Alle quali la patria poteva chiedere di sacrificare i propri figli.

Le componenti fondamentali della tragedia che attraverso l'esistenza delle madri che videro nella guerra il proseguimento il proseguimento del processo di costituzione della nazione e dell'identità italiana sono qui riassunte esemplarmente: dalla muta rivolta provata al momento della partenza di figli alla impossibilità di dar voce a questo sentimento, dal ripiegamento su se stesse al momento del lutto fino, dopo la vittoria dell'Italia e l'armistizio, alla difesa patriottica delle ragioni della guerra [D'Amelia 2005, 176].

Durante e dopo il conflitto, si ebbe senza dubbio un rilancio di una vera e propria «mitologia della madre sacrificale». Le madri italiane avevano ben pochi mezzi per cercare di impedire o, almeno, ritardare la partenza di un figlio, cercando di evitargli per qualche tempo l'invio al fronte. Ma 
nell'immaginario collettivo, nelle menti dei soldati, l'idea della propria madre era spesso l'ultimo rifugio, l'unica presenza salvifica. Anche sul piano simbolico, le donne dovevano vivere eroicamente il sacrificio a cui erano state chiamate. Così per le vedove, che potevano con la vedovanza acquisire sì una maggiore indipendenza, ma anche subire una possibile caduta nella miseria. Ma le vedove rappresentavano un caso potenzialmente bene più problematico. Le vedove non erano soltanto vittime, dovevano rendere conto della loro condotta, per non essere accusate di essere "vedove allegre", indegne della memoria del marito caduto [De Giorgio 1986].

Il lutto, la manifestazione pubblica del dolore, divenne uno dei modi, forse il principale, con cui la donna poteva contribuire alla mobilitazione generale per la guerra o, nel dopoguerra, per celebrare il sacrificio della patria e la memoria della vittoria; ma allo stesso tempo, un modo per rivendicare l'unicità del proprio dolore ${ }^{8}$. Pensiamo soltanto al Milite Ignoto, e in particolare a Maria Bergamas, che ebbe un figlio disperso in guerra, e a cui fu chiesto di scegliere, ad Aquileia, la salma che sarebbe stata tumulata sull'Altare della Patria [Tobia 1998].

\section{Manicomi}

In questa seconda parte dobbiamo appunto occuparci delle presenze femminili nei manicomi emiliano-romagnoli durante e dopo la Prima guerra mondiale. Raramente gli studi si sono soffermati su quanto la guerra con i traumi, le ansie e i lutti che provocò, e con la miseria che ne derivò, comportò per la salute psichica delle donne. Niente di confrontabile rispetto a quanto è stato scritto sulla follia dei militari ricoverati a migliaia durante il conflitto. Abbiamo cercato di indagare anche il ruolo avuto dalle donne nelle attività di cura e di assistenza ai ricoverati in manicomio. Occorre però anzitutto offrire un quadro sintetico delle struttura manicomiali esistenti in regione in quegli anni. Erano sei i manicomi pubblici esistenti [Paolella 2010; Tamburini, Ferrari e Antonini 1918]. A Reggio Emilia c'era il "San Lazzaro", un grande ospedale psichiatrico che accoglieva i malati provenienti anche dalla provincia di Modena e che già dagli ultimi 
decenni dell'Ottocento si era imposto come uno dei principali nel panorama nazionale, e in primo luogo come centro di ricerca. Poteva ospitare più di 1.500 degenti fra uomini e donne e durante il conflitto fu "invaso" da migliaia di militari inviati dal fronte perché presentavano i sintomi di una patologia nervosa o psichica'. Nelle province dell'Emilia occidentale esistevano anche i manicomi, ben più piccoli di quello reggiano, di Colorno (in provincia di Parma) e di Piacenza - ognuno dei quali poteva contenere circa 400 ricoverati.

L'altra grande struttura in regione era rappresentata dal manicomio di Imola, che aveva anche una succursale nella periferia cittadina e che ospitava i degenti provenienti da tutta la provincia bolognese. La città di Bologna aveva un proprio manicomio, il "Roncati", in pieno centro. Anche a Ferrara e sempre in quella città si instaurò nel 1915 l'ospedale militare neurologico "Villa del Seminario", creato da Gaetano Boschi, vice-direttore del manicomio provinciale [Raito 2010].

La guerra sconvolse direttamente anche la vita quotidiana degli ospedali psichiatrici, di chi vi era ricoverato come di chi vi lavorava. Fin dai primi mesi dall'inizio del conflitto, si verificò un sensibile incremento nel numero degli ingressi e, di conseguenza, nel numero dei ricoverati. Centinaia di militari traumatizzati, ammutoliti, immobili oppure clamorosi, giunsero anche nei manicomi della regione. E non va dimenticato che gli stessi manicomi in diversi casi dovettero "cedere" interi reparti, perché venissero trasformati in ospedali di riserva per soldati feriti. Ad esempio, il "San Lazzaro" di Reggio Emilia ebbe una quadruplicazione delle ammissioni complessive (uomini, donne, civili e militari) tra il 1915 (562 ammissioni) e il 1917 (2.059 ammissioni). L'anno successivo, il 1918, gli ingressi furono addirittura 3.273 , per poi iniziare a diminuire nel periodo successivo [Baraldi 1975] ${ }^{10}$. Il progressivo sovraffollamento al "San Lazzaro" influì senza dubbio - e non fu il solo caso nei manicomi italiani - nella diffusione di malattie contagiose molto pericolose. Nell'estate del 1915 al "San Lazzaro" comparve il colera, il che obbligò a isolare i padiglioni. Su questa epidemia, abbiamo a disposizione la testimonianza di Virginia Guicciardi Fiastri, moglie dell'allora direttore Giuseppe Guic-

Sempre a Reggio Emilia si installò verso la fine del 1917, dopo Caporetto, il Centro psichiatrico-militare di prima raccolta, diretto da Placido Consiglio, deputato a essere il luogo unico dove far confluire, tutti i soldati "impazziti", per osservarli e scovare possibili simulatori [Paolella 2008].

Si è calcolato che i militari che passarono per il "San Lazzaro" furono almeno 5.700. Purtroppo non disponiamo, invece, di dati sugli ingressi suddivisi fra uomini e donne. In generale, gli ingressi maschili sono sempre stati in maggior numero rispetto a quelli femminili. 
ciardi, nonché presidente del "Comitato Femminile di Assistenza civile":

In quel torno - metà settembre 1915 - si manifestarono a San Lazzaro fra le lavoranti del guardaroba (dove si raccoglievano le biancherie sudice dei soldati reduci dalla guerra) alcuni casi di colera. Dopo, altri casi all'“Agricola". Subito, per ordine governativo, S. Lazzaro resta chiuso in contumacia. Mio marito vorrebbe mandarmi a Reggio, ma io preferisco non lasciarlo. Debbo però sospendere... i miei rapporti col Comitato d'Assistenza a Reggio e coi miei due reparti di feriti che vengono chiusi. Ho preso il mio partito con grande serenità. Il colera fa strage e i portatori erano stati i feriti. Sono circa 23 casi al giorno. [...] A S. Lazzaro dopo 150 casi di colera e 50 morti, la vera guerra contro la terribile infezione combattuta strenuamente (a capo mio marito) con tutti i mezzi, alfine cessò [Virginia Guicciardi Fiastri, citata in Paterlini Brianti 2008, 207].

Passiamo al caso di Bologna. Qui abbiamo a disposizione dati più precisi. Il manicomio cittadino (intitolato a Francesco Roncati nel 1906) viveva già da molti anni prima della guerra problemi strutturali di sovraffollamento [Grillini 2011-2012]. Il che significava il dover patire una carenza sia di spazi utili sia di medici e infermieri. Come per tutti gli altri manicomi, lo scoppio della guerra comportò anche la partenza di una porzione importante del personale, soprattutto medico, che veniva arruolato nel Servizio neuro-psichiatrico dell'esercito.

Emergenze nuove che si aggiungevano ad altre, più antiche. La scarsità del personale riguardava anche il numero delle infermiere, così come il personale addetto alle cucine e alle pulizie. Ad esempio, al "Roncati" le infermiere in organico all'inizio della guerra erano soltanto 34 per tutto l'istituto. Dicevamo della crescita del numero dei ricoverati.

Le presenze nel manicomio bolognesi crebbero in modo lento, ma costante". Soffermiamoci sulle presenze mensili delle donne in manicomio. Un aumento significativo, simile a quello degli uomini, si ebbe in particolare nel corso del 1916. Se a gennaio del 1915 si trovavano al "Roncati" 288 donne (e 280 uomini), a dicembre dell'anno successivo erano 313 (contro 304 uomini). E sarebbero rimaste stabilmente oltre le 300 fino all'estate del 1919. Il numero delle dimissioni e delle morti in manicomio non subirono variazioni sostanziali. Con il dopoguerra anche a Bologna i ricoveri manicomiali iniziarono poi a ridursi, con un calo più marcato fra gli uomini. Senza dubbio, l'aumento degli ingressi femminili dipese dalla vita di guerra, e in primo luogo dalle difficoltà crescenti nella gestione di tante famiglie. In un contesto così difficile, era più facile che si dovesse 
ricorrere alla struttura manicomiale per contenere persone clamorose o pericolose a sé e agli altri. Paure, angosce per la partenza degli uomini, solitudine, difficoltà economiche, a volte miseria: tutto ciò non poteva che comportare un calo, a volte drammatico, della qualità della vita. Ma ciò nonostante - e come vedremo meglio più avanti - i medici non videro in questi problemi la causa reale del manifestarsi di tante patologie mentali, riconoscendo piuttosto l'emergere di malattie mentali in persone già comunque predisposte.

Passiamo ora a un manicomio molto più piccolo, quello di Colorno, oggetto di una importante ricerca, proprio sugli anni della Grande guerra, da parte di Ilaria La Fata ${ }^{12}$. Anche in questa struttura la guerra non fece che acuire i problemi di spazio e di gestione. I ricoverati crebbero in modo costante, passando dai 446 del 1914 ai 605 del 1918. Una gravissima mancanza di letti - in certi momenti si doveva far dormire per terra su semplici pagliericci - tale da trasformare quella che era stata la reggia ducale in un vero e proprio labirinto. Oltre ai nuovi ricoveri, ci fu - dopo Caporetto - da sistemare un gruppo nutrito di ricoverati (alcune decine) provenienti dai manicomi sfollati del Nord-est ${ }^{13}$.

Anche se la maggior parte dei ricoveri di questi anni interessò prevalentemente gli uomini - soprattutto soldati -, senza dubbio i problemi connessi alla crescente massa di degenti riguardarono pure le donne, che presto si trovarono a dover disporre di spazi sempre più angusti. Per questo motivo, nel corso del 1917, si rese necessario sistemare i reparti femminili delle "tranquille" e delle "agitate". Furono dunque riadattati i locali del secondo cortile del palazzo, e si costituì un nuovo reparto che poteva ospitare una quarantina di ricoverate, in prevalenza "semiagitate" [La Fata inedito].

Non abbiamo a disposizione - se non, talvolta, fra le lettere dei malati conservate nelle cartelle cliniche - un punto di vista diverso da quello dei medici, e del medico-direttore in particolare (che a Colorno durante la guerra era Ferdinando Ugolotti). La qualità della vita nel manicomio era severamente compromessa anche dalla scarsità del personale. Oltre a Ugolotti, erano in servizio soltanto altri due medici. Gli infermieri erano complessivamente 51 (di cui 24 donne), a cui si aggiungevano 4 suore ispettrici per i reparti femminili. Con il passare dei mesi, la direzione fu infine costretta ad assumere personale aggiuntivo, con contratti a termine.

Ringrazio Ilaria La Fata per avermi fatto leggere un suo saggio, ancora inedito, sulla Grande guerra nel manicomio di Colorno. 
Interessante ciò che riguardava la gestione del personale:

Le donne, in primo luogo quelle che già lavoravano in manicomio, parteciparono alle richieste di miglioramento nella stessa misura dei loro colleghi uomini. In concomitanza con l'aumento dei richiamati e con la difficoltà di trovare sostituti maschili, anche nel piccolo manicomio di Colorno il direttore aveva cominciato ad incrementare il personale femminile. Inizialmente le lavoratrici, nonostante il regolamento prevedesse una rigida divisione dei ruoli nei vari reparti e assegnasse loro mansioni quasi esclusive di pulizia dei locali e cura dei ricoverati, furono impiegate per svolgere anche ruoli più tradizionalmente maschili, come la sorveglianza nei reparti o la gestione di sartoria e cucine [La Fata inedito].

\section{Maria Del Rio e le donne del "San Lazzaro"}

Dagli esiti delle ricerche condotte direttamente sulle cartelle cliniche delle persone entrate in manicomio, emerge assai spesso una correlazione fra i ricoveri femminili - anche in particolare per il periodo che qui ci interessa - e la vita familiare. Le donne potevano essere inviate in manicomio per "raddrizzare" la condotta, pubblica o privata, di mogli, figlie, sorelle. Non si trattava soltanto di "marginali" (come prostitute e alcoliste) ${ }^{4}$. Famiglie "difficili" o semplicemente investite dalla guerra. Famiglie dove venivano a mancare all'improvviso uomini e denaro. Esistenze complicate, che potevano portare in manicomio, semmai per un breve periodo. Per parte sua, l'istituzione manicomiale non faceva che riconfermare, anche in queste condizioni eccezionali, la propria funzione di controllo sociale, ancora prima che di cura.

Come abbiamo detto, non esistono molti studi su questo argomento. Un silenzio che potrebbe essere vinto andando direttamente negli archivi clinici degli ex-ospedali psichiatrici.

La lettura delle cartelle cliniche di donne ricoverate in manicomio per cause riconducibili, direttamente o indirettamente, alla guerra rivela una realtà finora trascurata. [...] Per evitare che "anche la guerra, anche questa storia di uomini, contribuisca ad escludere, ad emarginare ancora di più la donna", è necessario dunque che con la lettura delle carte dei manicomi si apra un percorso di ricerca specifico 
sulle donne, poiché "bombardamenti, paure e fame sono soprattutto, prima di tutto, un affare di donne. Così le donne, tutte, hanno fatto anch'esse la guerra per molti anni", e non tutte sono riuscite a fronteggiarla mantenendo integro il proprio equilibrio psichico [Cremonini 1992, 83].

Maria Del Rio, era una delle poche psichiatre in quegli anni in Italia. La Del Rio venne assunta al "San Lazzaro" giusto nel 1915, anche perché nel manicomio reggiano si era reso necessario sostituire medici richiamati dall'esercito. Fu impegnata nel reparto femminile fino al 1921, quando venne incaricata di creare un reparto per l'assistenza e la cura di bambini con problemi intellettivi o di comportamento, la "Colonia Marro". Il nome della Del Rio è legato anche a una sua ricerca, pubblicata sulla "Rivista Sperimentale di Freniatria" già nel 1916 e dedicata a Le malattie mentali nella donna in rapporto alla guerra. Questo studio ci è utile anche perché riprende alcune fra le questioni più dibattute e delle idee più diffuse nel mondo psichiatrico italiano, sia sulla "follia femminile", sia più in generale sui danni prodotti dalla guerra sui nervi e sulle menti delle persone [Bianchi 2001; Gibelli 1998]. Occorre sottolineare anzitutto che la Del Rio scriveva nel 1916. La fine della guerra era ancora ben lontana (ed essa d'altra parte non tenne conto del fatto che non si poteva immaginare quanto il conflitto sarebbe durato e che piega avrebbe preso). La psichiatra reggiana selezionò una decina di casi di donne ricoverati al "San Lazzaro" all'indomani dello scoppio della guerra. All'inizio del saggio viene sottolineato un aumento nel numero di donne ricoverate.

Dall'inizio della guerra le ammissioni nel nostro Istituto sono aumentate di numero. Il maggior contingente dei nuovi ammessi è dato dagli uomini, specialmente dai soldati: da quelli cioè che più direttamente risentono degli effetti di questo terribile fenomeno sociale. Però, anche sulle donne, sebbene in proporzione assai minore, la guerra sembra aver avuto nel campo psichico la sua dolorosa ripercussione. Infatti, dal Giugno 1915 al Maggio 1916, le donne furono accolte nel nostro Istituto nella maggiore proporzione di circa il $12 \%$ rispetto alla media di un corrispondente periodo di tempo dei tre anni precedenti [Del Rio 1916, 87].

La guerra aggravò senza dubbio le condizioni economiche, alimentari, igieniche delle famiglie. La questione vera riguardava però capire quale responsabilità, anche indiretta, avessero le paure, le ansie, i lutti causati dalla guerra sull'insorgere di patologie mentali. Era la guerra la causa ultima? La Del Rio non può riconoscere che tutti i patemi e le tutte preoccupazioni, potevano certamente fiaccare il morale di tante donne. Riconosce quindi l'importanza delle emozioni come cause esogene nell'insorgere della malattia. 
In tempi normali un dolore psichico ugualmente intenso avrebbe forse potuto provocare simili episodî. Difficilmente però si può immaginare, all'infuori di un periodo di guerra, un insieme di circostanze, che sottoponga la donna a prolungate emozioni, a ripetute ansiose attese, a timori fondati, a tristi presentimenti, a continue rievocazioni di persone care in situazioni pericolose e tragiche [Del Rio $1916,106]$.

Dai casi ricostruiti dalla Del Rio non emergeva una condizione economica particolarmente grave e disastrosa. Per diverse pazienti l'unica causa apparente era appunto di origine patematica. Non erano d'altra parte presenti particolari malattie sessuali, né alcolismo o tbc, né tanto meno quelle ricoverate venivano da una gravidanza o da un periodo di allattamento - tutti elementi critici che potevano favorire, così si pensava, l'insorgere di patologie mentali. A pesare erano le ansie e i patemi, prolungati così a lungo durante la guerra. Ma - e questo è il punto centrale - per la Del Rio queste emozioni così pressanti non potevano essere comunque una causa sufficiente a scatenare una psicopatia. Se una donna, pur provata, cadeva nella depressione, nella melanconia $\mathrm{o}$, più raramente, nella demenza o nel delirio, ci doveva essere un'altra, più profonda, ragione. Doveva esserci in altre parole una causa endogena. Qui la Del Rio cedeva, per così dire, alla tesi, dominante nel mondo psichiatrico italiano, della «predisposizione ereditaria». Alla quale potevano aggiungersi semmai delle «modificazioni fisiologiche della vita sessuale». Ecco che le donne, come per altri versi i soldati che divenivano folli nelle trincee, erano persone «a più debole immunità psichica».

L'idea della guerra, con tutte le sue conseguenze, diventa quasi un'ossessione angosciosa a cui moltissimi sono sottoposti. Fra i tanti, alcuni, a più debole immunità psichica, reagiscono con una malattia mentale, che porta spesso l'impronta degli avvenimenti dell'epoca e rappresenta talora come l'esagerazione dello stato d'animo che l'ha preceduta [Del Rio 1916, 107].

Quei patemi facevano soltanto erompere una patologia già latente. Proprio come per i soldati, i disagi e i traumi potevano essere al massimo concause, cause occasionali o acceleratori di patologie che sarebbero comunque emerse. Le condizioni eccezionali della guerra determinavano semmai una maggiore intensità dei sintomi.

Gli psichiatri italiani, e in primo luogo quelli impegnati direttamente nella Sanità militare, finivano quindi per ridimensionare molto o per negare del tutto l'impatto patogeno dei traumi bellici. In sostanza finivano per ammalarsi coloro che, uomini e donne, non avevano le risorse nervose e psichiche per reagire in modo sano alle paure e alle privazioni. La guerra poteva essere così assolta. La "colpa" era piuttosto nascosta nel malato. 
Ma non è tutto. Sempre la Del Rio mette a confronto le "psicosi di guerra" (vere e presunte) dei militari e quelle femminili. Per la psichiatra reggiana la guerra per la donna era «solo un'immensa fonte di dolore» (da notare l'accostamento di due parole come solo e immensa). Tutto si risolveva soltanto in una lunga attesa, soltanto in una catena di rinunce, di pianti e di cattivi pensieri. Le donne, in fin dei conti, non vedevano la guerra "dal vivo", ne subivano solo indirettamente gli effetti. Tutto sommato, rimane dunque un sottofondo ottimista nel discorso della Del Rio: la guerra da sola molto improbabilmente avrebbe potuto condurre alla follia. Non si può accusare la guerra di aumentare da sola il numero delle malate di mente. Le si può forse attribuire, per il tramite della donna, una ripercussione sull'avvenire.

Le generazioni concepite negli anni di guerra pagheranno un maggior tributo alle malattie mentali, tarda manifestazione delle sofferenze e delle angosce subite dalle madri: ma, come per secoli e secoli è avvenuto, in un tempo più o meno lungo la stirpe umana si riavvicinerà al tipo normale da cui era stata deviata e la vita riprenderà vittoriosa il suo cammino ascensionale [Del Rio 1916, 108].

Anche qui campeggia quell'ottimismo di cui dicevamo. E campeggia il tentativo di ridurre la donna al ruolo "naturale" di madre, all'essenzialità assoluta della sua funzione riproduttiva.

\section{Bibliografia}

Audoin-Rouzeau S. e Becker A. 2002, La violenza, la crociata, il lutto. La Grande Guerra e la storia del Novecento, Torino: Einaudi.

Baraldi M. 1975, Statistica dal 1821 al 1974: movimento dei ricoverati e delle degenze negli Istituti Psichiatrici Ospedalieri Neuropsichiatrici del "San Lazzaro" di Reggio Emilia, Reggio Emilia: Age.

Bettiol N. 2008, Feriti nell'anima: storie di soldati dai manicomi del Veneto 1915-1918, Treviso: Istresco.

Bianchi B. 1983, Predisposizione, commozione o emozione? Natura e terapia delle neuropsicosi di guerra (1915-1918) in "Movimento operaio e socialista", 3.

Bianchi B. 1986, Delirio, smemoratezza e fuga, il soldato e la patologia della paura in Leoni D. e Zadra C. (eds) 1986, La Grande Guerra. Esperienza, memoria, immagini, Bologna: il Mulino.

Bianchi B. 2001, La follia e la fuga. Nevrosi di guerra, diserzione e disobbedienza nell'esercito italiano (1915-1918), Roma: Bulzoni.

Boschi G. 1931, La guerra e le arti sanitarie, Milano: Arnoldo Mondadori. 
Bracco B. 2012, La patria ferita: i corpi dei soldati italiani e la Grande guerra, Milano: Giunti.

Bravo A. 1980, Donne contadine e I guerra mondiale, "Società e storia", 10.

Cassata F. 2006, Molti, sani e forti. L'eugenetica in Italia, Torino: Bollati Boringhieri.

Ciceri M. 2009, Origini controllate. La prima eugenetica italiana (1900-1924), Civitavecchia: Prospettiva.

Cremonini S. 1992, Silenzio e solitudine di donne, in Sorcinelli P. (ed) 1992, La follia della guerra. Storie dal manicomio negli anni Quaranta, Milano: Franco Angeli.

Curli B. 1998, Italiane al lavoro 1914-1920, Venezia: Marsilio.

D’Amelia M. 2005, La mamma, Bologna: il Mulino.

De Giorgio M. 1986, Dalla "Donna nuova” alla donna della "nuova Italia”, in Leoni D. e Zadra C. (eds) 1986, La Grande Guerra. Esperienza, memoria, immagini, Bologna: il Mulino.

Del Rio M. 1916, Le malattie mentali della donna in rapporto alla guerra, "Rivista Sperimentale di Freniatria", 1.

Di Cori P. 1986, Il doppio sguardo, in Leoni D. e Zadra C. (eds) 1986, La Grande Guerra. Esperienza, memoria, immagini, Bologna: il Mulino.

Ermacora M. 2006, Le donne italiane nella Grande Guerra. Un bilancio storiografico (1990-2005), in Donne in guerra 1915-1918. La Grande Guerra attraverso l'analisi e le testimonianze di una terra di confine 2006, Tione di Trento: Centro Studi Judicaria.

Gibelli A. 1980, Guerra e follia. Potere psichiatrico e patologia del rifiuto nella grande guerra, "Movimento operaio e socialista", 4.

Gibelli A. 1986, L'esperienza di guerra. Fonti medico-psichiatriche e antropologiche in Leoni D. e Zadra C. (eds) 1986, La Grande Guerra. Esperienza, memoria, immagini, Bologna: il Mulino.

Gibelli A. 1998, L'officina della guerra. La Grande Guerra e le trasformazioni del mondo mentale, Torino: Bollati Boringhieri.

Gibelli A. 1998, La Grande Guerra degli italiani 1915-1918, Milano: Sansoni.

Gibelli A. 2005, La nazione in armi. Grande Guerra e organizzazione del consenso, in De Luna G., D'Autilia G. e Criscenti L. (eds) 2005, L'Italia del Novecento. Le fotografie e la storia. I. Il potere da Giolitti a Mussolini (1900-1945), Torino: Einaudi.

Grillini A. 2011-2012, Il manicomio di guerra. L'ospedale psichiatrico "Francesco Roncati” di Bologna tra il 1915 e il 1920, Tesi di laurea in Scienze storiche e forme della memoria, Università degli studi di Trento.

Labita V. 1986, La psicologia militare italiana (1915-1918) in Leoni D. e Zadra C. (eds) 1986, La Grande Guerra. Esperienza, memoria, immagini, Bologna: il Mulino.

La Fata I. inedito, Una comunità ai margini. Militari e civili nel manicomio di Colorno (Parma).

Lussu E. 1960, Un anno sull'altopiano, Torino: Einaudi. 
Mantovani C. 2004, Rigenerare la società: l'eugenetica in Italia dalle origini ottocentesche agli anni Trenta, Soveria Mannelli: Rubbettino.

Molinari A. 2001, Appunti per una storia delle donne nella Grande Guerra, "Quaderni del Dipartimento di lingue e letterature straniere moderne", 11.

Molinari A. 2008, Donne e ruoli femminili nell'Italia della Grande Guerra, Milano: Selene.

Paolella F. 2008, Un laboratorio di medicina politica. Placido Consiglio e il Centro psichiatrico militare di prima raccolta, in Carrattieri M. e Ferraboschi A. (eds) 1997, Piccola patria Grande guerra. La Prima Guerra Mondiale a Reggio Emilia, Bologna: Clueb.

Paolella F. 2010, La neuro-psichiatria in Emilia-Romagna durante la Grande Guerra, in Montella F., Paolella F. e Ratti F. (eds) 2010, Una regione ospedale. Medicina e sanità in Emilia-Romagna durante la Prima Guerra Mondiale, Bologna: Clueb.

Paolella F. 2013, Violenze sessuali nella prima guerra mondiale. Medicina e politica in Italia in "Storia e problemi contemporanei", 62.

Paterlini Brianti E. 2008, La mobilitazione femminile. Le donne reggiane e le associazioni di volontariato civile durante la Grande Guerra, in Carrattieri M. e Ferraboschi A. (eds) 1997, Piccola patria Grande guerra. La Prima Guerra Mondiale a Reggio Emilia, Bologna: Clueb.

Pogliano C. 1984, Scienza e stirpe: eugenica in Italia (1912-1939) in "Passato e presente", 5.

Raito L. 2010, Gaetano Boschi. Sviluppi della neuropsichiatria di guerra (1915-1918), Roma: Carocci.

Scartabellati A. 2003, Intellettuali nel conflitto. Alienisti e patologie attraverso la Grande Guerra (1909-1921), Bagnaria Arsa: Goliardica.

Scartabellati A. 2008, Dalle trincee al manicomio. Esperienza bellica e destino di matti e psichiatri nella Grande Guerra, Torino: Marco Valerio.

Schwarz G. 2010, Tu mi devi seppellir. Riti funebri e culto nazionale alle origini della Repubblica, Torino: Utet.

Soldani S. 1991, Donne senza pace. Esperienze di lavoro, di lotta, di vita tra guerra e dopoguerra (1915-1920), “Annali dell'Istituto Alcide Cervi”, 13.

Tamburini A. 1916, L'organizzazione del servizio nevro-psichiatrico di guerra nel nostro Esercito, "Rivista Sperimentale di Freniatria", 2.

Tamburini A., Ferrari G. C. e Antonini G., L'assistenza degli alienati in Italia e nelle varie nazioni, Torino: Utet.

Thébaud F. 1992, La Grande Guerra: età della donna o trionfo della differenza sessuale?, in Duby G., Perrot M. (eds) 1992, Storia delle donne in Occidente. Il Novecento, Roma-Bari:Laterza.

Tobia B. 1998, L'Altare della Patria, Bologna: il Mulino.

Willson P. 2010, Italiane. Biografia del Novecento, Roma-Bari: Laterza 
Winter J. 1998, Il lutto e la memoria. La Grande guerra nella storia culturale europea, Bologna: il Mulino. 